\title{
Completion of the 8 MW Multi-Frequency ECRH System at ASDEX Upgrade
}

D. Wagner ${ }^{\mathrm{a}}$, J. Stober ${ }^{\mathrm{a}}$, M. Kircher ${ }^{\mathrm{a}}$, F. Leuterer ${ }^{\mathrm{a}}$, F. Monaco ${ }^{\mathrm{a}}$, M. Münich ${ }^{\mathrm{a}}$, M. Schubert ${ }^{\mathrm{a}}$, H. Zohm ${ }^{\mathrm{a}}$,

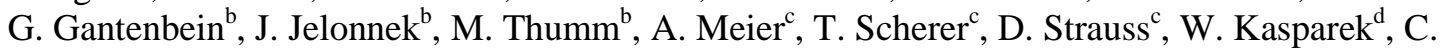

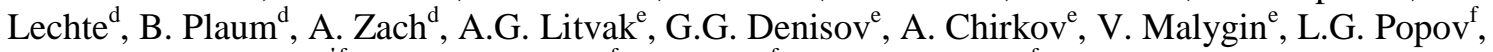
V.O. Nichiporenko ${ }^{\dagger f}$, V.E. Myasnikov ${ }^{f}$, E.M. Tai ${ }^{f}$, E.A. Solyanova ${ }^{\mathrm{f}}$ and ASDEX Upgrade team

${ }^{a}$ Max-Planck-Institut für Plasmaphysik, Boltzmannstr.2, D-85748 Garching, Germany

${ }^{\mathrm{b}}$ Institut für Hochleistungsimpuls- und Mikrowellentechnik, ${ }^{\mathrm{C}}$ Institut für Angewandte Materialien-AWP, Karlsruhe Institute of Technology, Kaiserstr. 12, D-76131 Karlsruhe, Germany

${ }^{\mathrm{d}}$ Institut für Grenzflächenverfahrenstechnik und Plasmatechnologie, Universität Stuttgart, Pfaffenwaldring 31, D-70569 Stuttgart, Germany

${ }^{\mathrm{e}}$ Institute of Applied Physics, RAS, 46 Ulyanov St., Nizhny Novgorod, 603950, Russia

${ }^{\mathrm{f}}$ GYCOM Ltd, 46 Ulyanov St., Nizhny Novgorod, 603155, Russia

\begin{abstract}
Over the last 15 years, the Electron Cyclotron Resonance Heating (ECRH) system at the ASDEX Upgrade tokamak has been upgraded from a $2 \mathrm{MW}, 2 \mathrm{~s}, 140 \mathrm{GHz}$ system to an $8 \mathrm{MW}, 10 \mathrm{~s}$, dual frequency system $(105 / 140 \mathrm{GHz})$. Eight gyrotrons were in routine operation during the current experimental campaign. All gyrotrons are step-tunable operating at 105 and $140 \mathrm{GHz}$ with a maximum output power of about 1 MW and $10 \mathrm{~s}$ pulse length. The system includes 8 transmission lines, mainly consisting of oversized corrugated waveguides (I.D. $=87 \mathrm{~mm}$ ) with overall lengths between 50 and 70 meters including quasi-optical sections at both ends. Further improvements of the transmission lines with respect to power handling and reliability are underway.
\end{abstract}

\section{INTRODUCTION}

A FTER successful completion of the ECRH system, 8 gyrotrons are now in routine operation at ASDEX Upgrade. All gyrotrons are step-tunable working at both, 105 and $140 \mathrm{GHz}$ with output powers up to $1 \mathrm{MW}$ at $140 \mathrm{GHz}$ and slightly reduced output power at $105 \mathrm{GHz}$ with a maximum pulse length of $10 \mathrm{~s}$, corresponding to the maximum flat top pulse length of the tokamak. The gyrotrons are equipped with depressed collectors and their efficiencies are around $50 \%$. More than $5 \mathrm{MW}$ of ECRH power has been successfully coupled to the plasma during the current experimental campaign. The installed ECRH power now exceeds the $\mathrm{L} / \mathrm{H}$-mode threshold in ASDEX Upgrade at least by a factor of two even for high electron densities and is comparable to the installed power of the low frequency Ion Cyclotron Heating System. The power of both RF heating systems (>10 MW) is about half of the power of the Neutral Beam Injection system. This leads to a high flexibility for the plasma experiments with respect to torque input, shape of heating profile and variation of the ratio between electron and ion heat flux $\left(\mathrm{Q}_{\mathrm{e}} / \mathrm{Q}_{\mathrm{i}}\right)$ even at high heating power. The frequency tunability of the gyrotrons increases the operating range of the system with respect to the magnetic field. After completion of the ECRH system the main focus is now on the further improvement of its reliability and the power handling capability of the transmission lines.

\section{ECRH TRANSMISSION LINE}

Fig.1 shows the one of the two gyrotron installations at ASDEX Upgrade with 4 gyrotrons each (ECRH2 and ECRH3). The overall lengths of the transmission lines are about $50 \mathrm{~m}(\mathrm{ECRH} 2)$ and $70 \mathrm{~m}(\mathrm{ECRH} 3)$. The gyrotrons are connected to a quasi-optical Matching Optics Unit (MOU), which is used for beam conditioning and adjustment of the polarization (Fig.2). It contains an individual pair of phase correcting mirrors for each frequency, which are also used to align the beam with respect to a common beam axis for both frequencies. The phase correcting mirrors are followed by a broadband universal polarizer consisting of two grooved mirrors [2]. The fifth mirror focuses the beam into the input of the corrugated waveguide section. For gyrotron conditioning the beam can be alternatively directed into a $1 \mathrm{~s}, 1 \mathrm{MW}$ water load, which is part of each MOU. There is also one long-pulse (1 MW, 10s) and one short-pulse (200 ms) calorimetric load installed in both ECRH2 and ECRH3 installations. A quasioptical mirror box at the end of each transmission line allows for switching the beam either to the torus vacuum window or to a second calorimetric load installed next to the torus (Fig.3). This load can be used to measure the transmission efficiency and for conditioning of the transmission line.

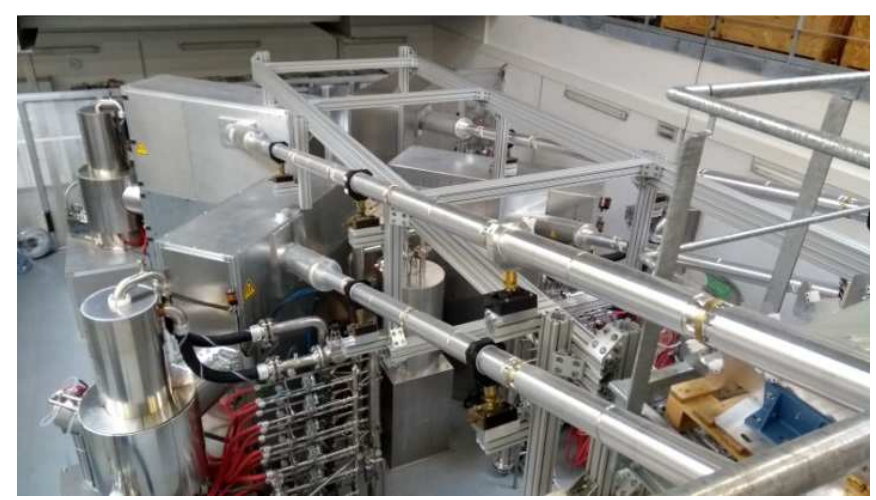

Fig. 1. Gyrotron installation ECRH3 at ASDEX Upgrade.

\section{RELIABILITY AND POWER HANDLING IMPROVEMENTS}

Since the transmission lines are operating under atmospheric pressure, occasional arcing turned out to be a problem at power levels approaching the maximum output power of the gyrotrons. The arc detection safety system is based on photo diodes that are connected to different locations along the transmission line via optical fibers. The speed of this interlock depends on the location where arcing occurs. Arcs 
that are not immediately "seen" by the system can strongly

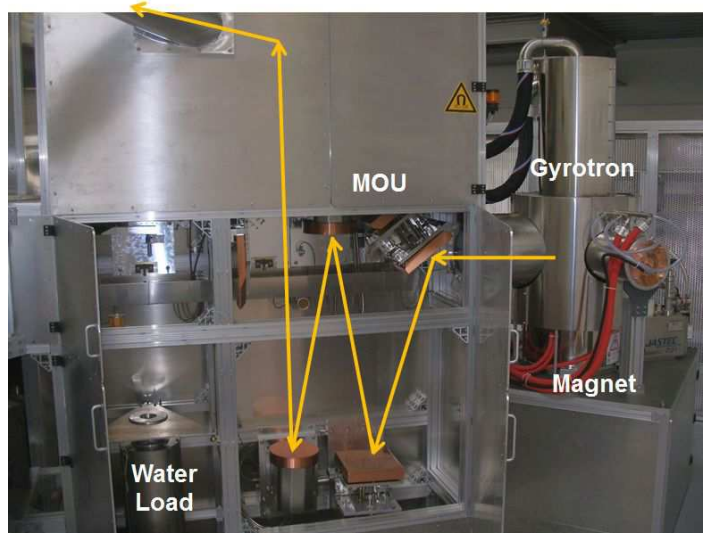

Fig. 2. Matching Optics Unit (MOU).

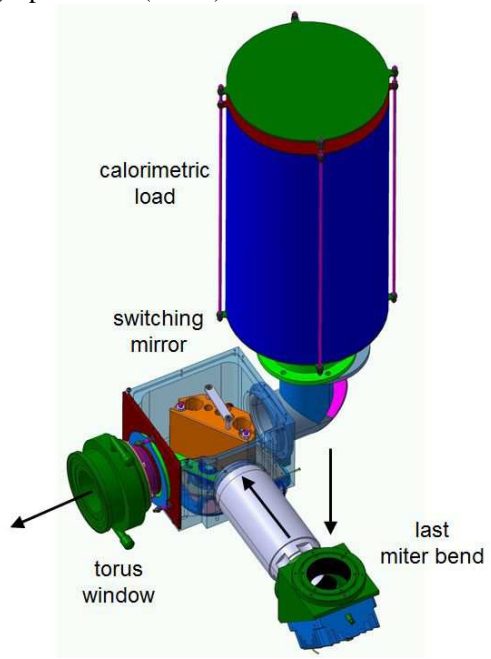

Fig. 3. Quasi-optical mirror box at the end of the waveguide transmission line The output beam can be switched to the torus (arrows) or to the calorimeter.

increase the contents of radicals in the transmission line which leads to further deconditioning. On some of the transmission lines an additional fast interlock for arcing was recently added by combining power signals from directional couplers in the second phase correcting mirror in the Matching Optics Unit (MOU) and the last miter bend mirror at the end of each transmission line. Since the polarizers are located in-between the two directional couplers, the power measurement in the last miter bend was polarization dependent. To overcome this, a second directional coupler with perpendicular orientation was integrated into this miter bend mirror where one coupler is oriented for E-plane and the other one for H-plane coupling (Fig.4). Therefore, the combination of both signals becomes independent of the polarization. Fast arc detection is provided by comparing the power signal at the end of the transmission line with the one from in the MOU. As a further measure against arcing a ventilation system was installed to flush the transmission lines with dry air. For this a ring of small holes with diameters below cutoff was introduced in a waveguide approximately in the middle of the transmission line (Fig.5). A Shutter was installed between the large volume of the MOU and the waveguide transmission line. A small flow of dry air of $1.25 \mathrm{l} / \mathrm{min}$ is permanently supplied to the waveguide, mainly to prevent humid air from entering the waveguide atmosphere. After the occurrence of an arc, the waveguide can be flushed with $5 \mathrm{~m}^{3} / \mathrm{h}$ (corresponding a flow of dry air with $0.24 \mathrm{~m} / \mathrm{s}$ ) to remove radicals produced during the arc and the high-power transmission. One of the transmission lines showed severe deconditioning in the past. It allowed only for the transmission of a maximum power of about $600 \mathrm{~kW}$ without arcing. A vertical section near the end of the transmission line showed oxidation of the corrugated inner surface of the aluminum waveguides. Small aluminum oxide dust particles could be found on the miter bend mirror below this vertical waveguide section. This further increases the probability of arcing. To prevent this, the waveguide surface was sealed by chromium plating of the waveguides in this section. Additionally the dry air ventilation system was automated for this transmission line, where the shutter to the MOU is opened just $3 \mathrm{~s}$ before the gyrotron pulse and the waveguides are flushed with $5 \mathrm{~m}^{3} / \mathrm{h}$ and this strong flush is continued until $30 \mathrm{~s}$ after the pulse before the shutter is closed and the ventilation is reduced to the low, permanent flow. All three measures against arcing were tested at one specific transmission line. The transmitted power could be efficiently increased from 650 to $880 \mathrm{~kW}$, corresponding to the maximum output power of the installed gyrotron. Similar improvements at all other transmission lines are underway.
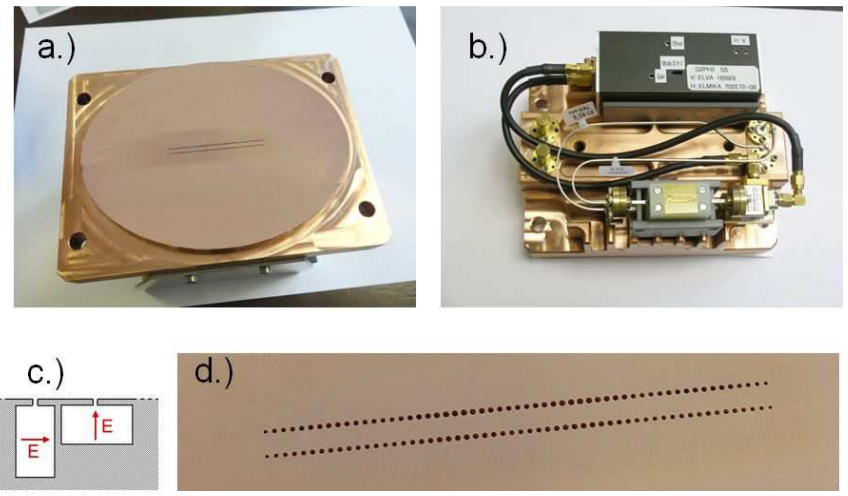

Fig. 4. Polarization independent miter bend power detector.
a.) Front side
b.) Rear side
c.) Built-in waveguide orientation
d.) Close-up of the coupling hole arrays
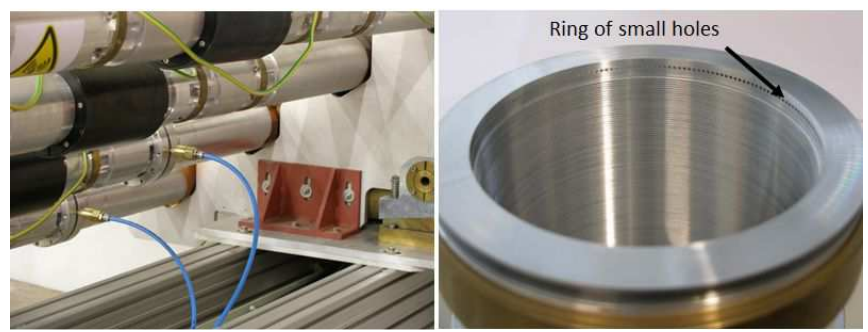

Fig. 5. Waveguide ventilation system. Dry air supply mounted on waveguide flanges (left), ring of cutoff holes at the end of a corrugated waveguide (right).

\section{REFERENCES}

[1]. D. Wagner et al., "Status, Operation and Extension of the ECRH System at ASDEX Upgrade", Journal of Infrared, Millimeter and Terahertz Waves, vol. 37, pp. 45-54, 2016.

[2]. D. Wagner, F. Leuterer, "Broadband polarizers for high power multifrequency ECRH systems", Journal of Infrared, Millimeter and Terahertz Waves, vol. 26, 163-172, 2005. 